\title{
PRETEXT Staging System
}

National Cancer Institute

\section{Source}

National Cancer Institute. PRETEXT Staging System. NCI Thesaurus. Code C141133.

A staging system designed by the International Childhood Liver T umor Strategy Group (SIOPEL) for staging and risk stratification of liver tumors. A PRET EXT stage number is based on the number of contiguous liver sections not involved by tumor, corresponding to a rough estimate of the difficulty of the expected surgical procedure. It is used to describe tumor extent before any therapy, thus allowing more effective comparison between studies conducted by different groups. 